\title{
Spectral Imaging with Near-Field Infrared Spectroscopy and Microscopy
}

\author{
C. A. Michaels*, D. B. Chase** and S. J. Stranick* \\ * National Institute of Standards and Technology, 100 Bureau Drive, Gaithersburg, MD 20899 \\ ** DuPont Central Research and Development, Wilmington, DE 19880
}

The ability to measure chemical bond changes on the nanometer scale is of critical importance for the characterization of surfaces relevant to catalysis, materials and biological problems. Our goal has been to develop a technique for the in-situ, non-destructive, nanoscale chemical imaging of surfaces. The strategy for realizing this goal involves coupling the high spatial resolution of near-field scanning optical microscopy (NSOM) [1,2] with the chemical specificity of vibrational spectroscopy [3]. The combination of the sub-diffraction spatial resolution attainable in the near-field with the high chemical specificity of infrared absorption spectroscopy promises a powerful new analytical instrument that overcomes critical measurement limitations of both far-field infrared microscopes (low spatial resolution) and scanned probe microscopes (lack of chemical specificity).

There are a number of challenges that must be met to make near-field infrared absorption microscopy/ spectroscopy a reality. Particularly, near-field probes compatible with infrared radiation and a bright, tunable infrared source are required. We have developed a novel bench-top infrared NSOM instrument that incorporates tapered, fluoride glass fiber probes and a tunable, broadband, infrared laser source. A detailed description of the instrumental design and characterization of the instrumental sensitivity and spatial resolution has been reported [4].

Initial applications of this technique have been focused on measuring lateral variations in chemical composition for thin organic films such as polystyrene (PS)/polyethylacrylate (PEA) polymer blends, a model system for the study of degradation and corrosion of organic coatings. Characterization of lateral phase segregation in this system by near-field spectral imaging on the aromatic C-H stretching band of PS has been completed. Figure 1(a) shows the far-field IR absorbance spectra of PS (solid line) and PEA (dashed line) in the $\mathrm{CH}$ stretching region along with the spectrum of the broadband IR laser (gray) tuned to the center wavelength $(\lambda=3.36 \mu \mathrm{m}$ ) used to acquire this data. The obvious differences in absorbance over this spectral region, including distinct bands of PS absorbance (3025 $\left.\mathrm{cm}^{-1}\right)$ and PEA absorbance $\left(2980 \mathrm{~cm}^{-1}\right)$, allow for the differentiation of these two materials with spatial resolution $(\sim 400 \mathrm{~nm})$ well below the diffraction limit. Figure 1(b) shows an $8 \times 8 \mu \mathrm{m}$ nearfield spectral image frame of the PS/PEA film acquired at $2980 \mathrm{~cm}^{-1}$. Analysis of the spatial and spectral variations in near-field transmission contrast over many image frames allows for the identification of the minority phase (bright features) composition as being PS rich, in agreement with the results of a selective etching/ AFM analysis of a similar sample. This identification rests on the capability of this microscope to measure the differential absorbance due to a PS feature having only a nominal lateral size of $1 \mu \mathrm{m}^{2}$ and a thickness of $500 \mathrm{~nm}$. The broadband nature of the light source also allows for the analysis of the relative importance of near-field coupling and absorption as sources of near-field image contrast.

Figure 2 is another example of the types of data that can be extracted from a single near-field spectral image acquisition for these types of samples. Shown are 8 x $8 \mu \mathrm{m}$ near-field absorbance 
(a)

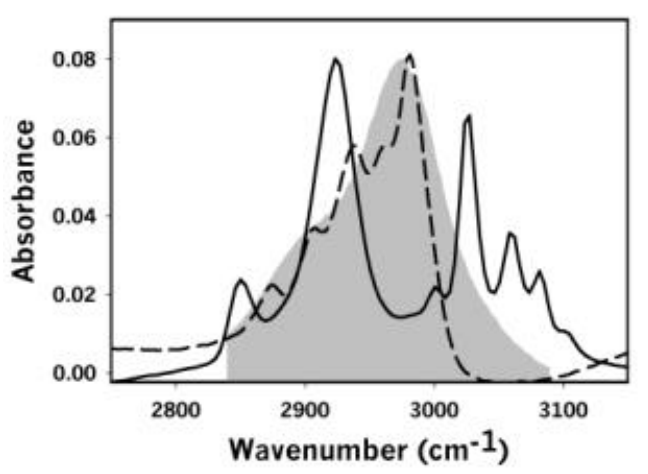

(b)

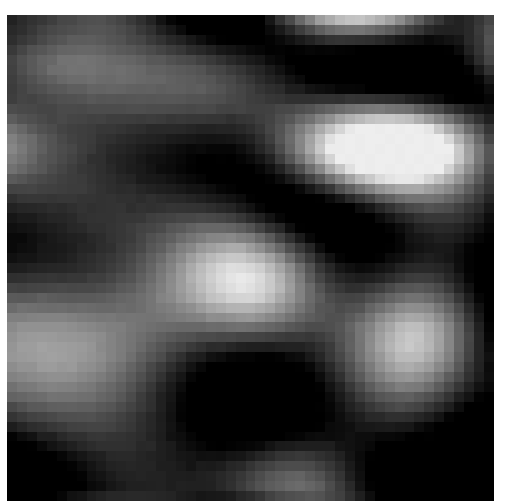

Figure 1. (a) Far-field absorbance spectra of PS (solid line) and PEA (dashed line) along with laser spectrum (gray) used to acquire near-field IR transmission images of a thin PS/PEA blend film. (b) $8 \mathrm{x}$ $8 \mu \mathrm{m}$ near-field transmission image of PS/PEA blend film acquired at $2980 \mathrm{~cm}^{-1}$.

images of a $1.1 \mu \mathrm{m}$ polystyrene microsphere in a polymethylmethacrylate (PMMA) film. Figure 2(a) is an image corresponding to an aromatic C-H stretching resonance of $\mathrm{PS}$ at $3120 \mathrm{~cm}^{-1}$ whereas Figure 2(b) is an image corresponding to an off-resonance frequency of $2970 \mathrm{~cm}^{-1}$. These images clearly demonstrate the high spatial resolution, chemical discrimination that is possible with this technique.

(a)

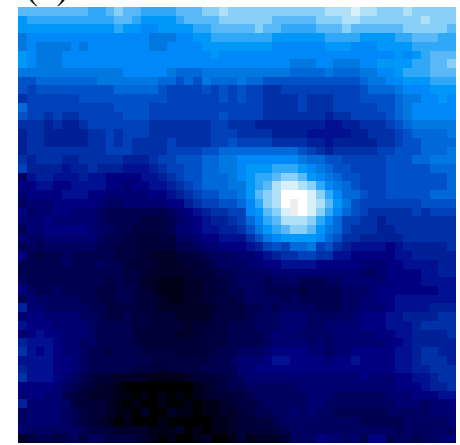

(b)

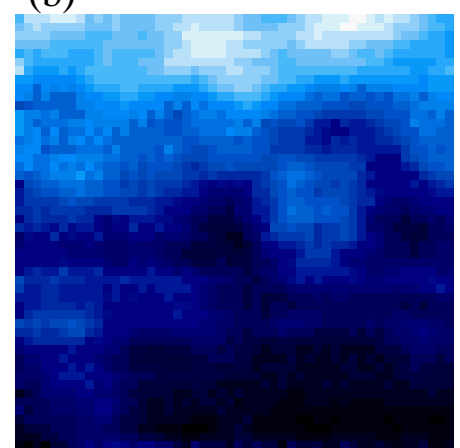

Figure 2. (a) $8 \times 8 \mu \mathrm{m}$ near-field absorbance image of a $1.1 \mu \mathrm{m}$ PS microsphere in a PMMA film acquired on a PS resonance at $3120 \mathrm{~cm}^{-1}$. (b) $8 \times 8 \mu \mathrm{m}$ near-field absorbance image (measured simultaneously with Figure 2(a)) of a $1.1 \mu \mathrm{m}$ PS microsphere in a PMMA film acquired off resonance at $2970 \mathrm{~cm}^{-1}$.

Future instrumental development efforts will focus on the use of apertureless probes as a means of achieving high spatial confinement of the infrared radiation, leading to further improvements in spatial resolution.

\section{References}

[1] D.W. Pohl et al., Appl. Phys. Lett. 44 (1984) 651.

[2] A. Lewis et al., Ultramicroscopy 13 (1984) 227.

[3] Y.T. Chabal et al., Vibrations at Surfaces, Elsevier, Amsterdam, 1990.

[4] C.A. Michaels, et al., J. Appl. Phys. 88 (2000) 4832. 